\title{
Escala diagramática para avaliação da severidade da Mancha Ocular em milho causada por Kabatiella zeae
}

\author{
Diagramatic scale for assessing severity of eyespot in maize caused by Kabatiella zeae
}

\author{
Rubia Cristiani Camochena ${ }^{I}$ Idalmir dos Santos $^{I *}$ Sergio Miguel Mazaro ${ }^{I}$
}

\section{RESUMO}

\begin{abstract}
O objetivo deste trabalho foi desenvolver uma escala diagramática para avaliação da severidade da mancha ocular, causada por Kabatiella zeae, em folhas de milho, considerando os limites de severidade mínima e máxima da doença e os níveis intermediários. Os níveis de severidade observados em campo seguiram incrementos logarítmicos, obedecendo a "Lei do Estímulo de Weber-Fechner”, representando: 0,9; 2,0; 4,0; 9,0; 18,0; 32,0 e 51,0\% da área foliar lesionada. A validação da escala consistiu-se em duas etapas: na primeira, 10 avaliadores, com experiência na avaliação de doenças em plantas, avaliaram 30 folhas com diferentes níveis de severidade, com e sem o uso da escala diagramática. Na segunda etapa, outros 10 avaliadores, sem experiência na avaliação de doenças, fizeram as mesmas avaliações, com e sem uso da escala diagramática. Por meio de regressão linear, foram confrontados valores de severidade reais e estimados e foram analisadas a acurácia e a precisão dos avaliadores. Constatou-se melhora na acurácia e na precisão das estimativas visuais efetuadas com o auxílio da escala diagramática. A escala diagramática proposta foi considerada adequada para estimar a severidade da mancha ocular em milho, podendo ser utilizada em estudos epidemiológicos para avaliação de reação de genótipos à doença e para implementação de estratégias de controle da doença.
\end{abstract}

Palavras-chave: validação, patometria, Zea mays.

\section{ABSTRACT}

The objective of this research was to develop a diagrammatic scale to assess the severity of Eyespot, caused by Kabatiella zeae in corn leaves, considering the limits for minimum and maximum severity of the disease, as well as intermediate levels. The levels observed in the field followed logarithmic increments, obeying “Weber-Fechner's StimulusResponse Law”, representing: 0.9; 2.0; 4.0; 9.0; 18.0; 32.0; and $51.0 \%$ injured leaf area. Scale validation consisted of two steps: in the first, 10 evaluators, experienced in the evaluation of diseases, evaluated 30 leaves with different levels of severity, with or without using the diagrammatic scale. In the second to evaluate, 10 other evaluators, without experience in the evaluation of diseases, performed the same evaluation, with or without using the diagrammatic scale. By linear regression, actual and estimated severity values were compared, and evaluator accuracy and precision was assessed. There was an improvement in the accuracy and precision of estimates made using the diagrammatic scale as a visual aid. The diagrammatic scale proposed was considered appropriate to estimate the severity of corn eyespot and can be used in epidemiological studies, to evaluate the reaction of genotypes to the disease, and to improve control strategies against the disease.

Key words: validation, pathometry, Zea mays.

\section{INTRODUÇÃO}

Entre as doenças que ocorrem na cultura do milho, foi constatada, recentemente, no Sul do Brasil, a mancha ocular causada por Kabatiella zeae Narita \& Hiratsuka [Sin.= Aureobasidium zeae (Narita \& Hiratsuka) Dingley]. A doença foi diagnosticada em dezembro de 2004, em folhas do híbrido de milho AG 9020, procedente dos municípios de Campo-Erê, Santa Catarina (SC) e de Palmas, Paraná (PR) (SANTOS et al., 2007). Nas safras subseqüentes, a mancha ocular assumiu maior importância, sendo detectada em mais de 30 genótipos de milho, na região sudoeste do Paraná, com severidades variando entre sete a $17 \%$ (CAMOCHENA et al., 2007).

'Universidade Tecnológica Federal do Paraná (UTFPR), Campus Pato Branco, PR 469, km 1, CP 571, 85501-970, Pato Branco, PR, Brasil. E-mail: idalmir@utfpr.edu.br. *Autor para correspondência. 
Os sintomas iniciais consistem de lesões encharcadas e translúcidas, circulares a ovais (1-4mm de diâmetro), com um halo amarelado, que, em seguida, tomam cor parda a creme no centro, ficando com aparência semelhante a um olho. Algumas manchas podem coalescer, formando grandes áreas necróticas e podendo produzir uma seca antecipada das folhas (REIS \& CASA, 2004; SANTOS et al., 2007).

A quantificação dos danos é o passo inicial para o desenvolvimento de qualquer programa de manejo de doenças. Como a mancha ocular vem crescendo em importância, por manifestar-se em vários híbridos de milho, os danos poderão se tornar expressivos. Apesar disso, não há estudos epidemiológicos no Brasil e, para que esses estudos sejam realizados, é necessário o desenvolvimento de métodos padronizados de quantificação da severidade da doença em campo. Sendo assim, é importante o uso de métodos de quantificação que sejam confiáveis e facilmente reproduzíveis para auxiliar o avaliador e minimizar a subjetividade da estimativa. Dessa forma, escalas diagramáticas têm sido uma ferramenta bastante útil (HORSFALL \& COWLING, 1978; BERGAMIN FILHO \& AMORIN, 1996; LEITE \& AMORIM, 2002; SPÓSITO et al., 2004).

Apesar da crescente importância da mancha ocular na agricultura brasileira, pouco se sabe sobre esse patossistema nestas condições. A elaboração e a validação de uma escala diagramática, para avaliação da severidade desta doença, constituem-se em uma ferramenta necessária em estudos que visam compreender a doença sob a influência de fatores ambientais e os níveis de resistência do hospedeiro e seu controle.

Para algumas doenças do milho já foram elaboradas escalas diagramáticas para quantificação da severidade, como as escalas para avaliação de mancha de feosféria e ferrugem do milho, que auxiliam em muitas pesquisas relacionadas à quantificação de doenças (AGROCERES, 1996). No entanto, em relação à mancha ocular, nada foi encontrado na literatura.

Assim sendo, considerando a inexistência de métodos para quantificação da mancha ocular em milho, este trabalho teve como objetivo desenvolver uma escala diagramática para avaliação da severidade dessa doença.

\section{MATERIAL E MÉTODOS}

Para elaboração da escala diagramática, foram colhidas 110 folhas de milho cultivado na área experimental da Universidade Tecnológica Federal do
Paraná - Campus Pato Branco, PR, na safra 2006/2007. Essas folhas apresentavam ampla variação de severidade da doença, sendo utilizada, para confecção da escala, uma amostra de $20 \mathrm{~cm}$ de comprimento da folha, descartando-se $20 \mathrm{~cm}$ da extremidade. A porção foi assim padronizada por ser difícil trabalhar com a folha inteira para reproduzir as lesões na escala. Além disso, a parte selecionada correspondeu à área da folha onde houve maior manifestação da doença (área representativa da doença). As áreas lesionadas foram copiadas em lâminas de transparência e suas dimensões, bem como as dimensões das amostras de folhas, foram determinadas por meio do medidor de área foliar, marca LI-COR, modelo 3100. Foram consideradas a máxima e a mínima proporção de área foliar lesionada como sendo os valores máximo e mínimo da escala diagramática. Os quatro níveis intermediários foram definidos matematicamente, seguindo incremento logarítmico e respeitando-se a acuidade da visão humana de acordo com a "Lei do estímulo de Weber e Fechner” (HORSFALL \& BARRATT, 1945).

Uma vez definidas as percentagens de área foliar lesionadas e seus correspondentes níveis a serem adotados na escala, foram obtidas, entre as $\mathbf{1 1 0}$ folhas iniciais, as que apresentavam severidade semelhante aos níveis indicados nos cálculos matemáticos. Então, com auxílio de máquina fotográfica digital e programa de edição de imagem (Adobe Photoshop 8.0), foi constituída a escala proposta.

Para validação da escala diagramática, foram tomadas 30 folhas com diferentes níveis de severidade e apresentadas a 20 avaliadores, sendo $10 \mathrm{com}$ experiência prévia e 10 sem experiência em avaliação de doenças em plantas[Com experiência: avaliador 1(A,K); 2(B,L); 3(C,M); 4(D,N); 5(E,O); 6(F,P); 7 (G,Q), 8(H,R); 9 (I,S); 10 (J,T), sem auxílio (A-J) e com auxílio (K-T)], [Sem experiência: avaliador $1\left(\mathrm{~A}^{*}, \mathrm{~K}^{*}\right) ; 2\left(\mathrm{~B}^{*}, \mathrm{~L}^{*}\right)$; 3(C*,M*); 4(D*,N*); 5(E*,O*); 6(F*,P*); $7\left(\mathrm{G}^{*}, \mathrm{Q}^{*}\right), 8$ $\left(\mathrm{H}^{*}, \mathrm{R}^{*}\right) ; 9\left(\mathrm{I}^{*}, \mathrm{~S}^{*}\right) ; 10\left(\mathrm{~J}^{*}, \mathrm{~T}^{*}\right)$, sem auxílio $\left(\mathrm{A}^{*} \mathrm{~J}^{*}\right)$ e com auxílio $\left.\left(\mathrm{K}^{*}-\mathrm{T}^{*}\right)\right]$, os quais estimaram, visualmente, a severidade da mancha ocular numa primeira etapa sem e, posteriormente, com o uso da escala diagramática. A porcentagem de área foliar afetada foi determinada considerando-se o halo encharcado da mancha, observando a folha contra a luz.

A acurácia e a precisão das estimativas visuais de cada avaliador foram determinadas por regressão linear simples, considerando severidade real (porcentagem de área foliar lesionada, determinada por incrementos logarítmicos) como variável independente e a severidade estimada como variável dependente. A precisão das estimativas foi avaliada pelo coeficiente de determinação da regressão $\left(\mathrm{r}^{2}\right)$ e pela variância dos 
erros absolutos (severidade estimada menos severidade real).

\section{RESULTADOS E DISCUSSÃO}

A escala diagramática proposta neste trabalho, com sete níveis de severidade $(0,9 ; 2,0 ; 4,0$; 9,0; 18,0; 32,0 e 51,0\%), observou a "Lei do estímulo de Weber-Fechner”, conforme descrita em trabalhos anteriores de elaboração de escalas (HORSFALL \& COWLING, 1978; RODRIGUES et al., 2002; GOMES et al., 2004; MAZARO et al., 2006), que pode ser observada na figura 1.

Na validação da escala diagramática, os avaliadores mostraram-se com boa acurácia, pois os valores estimados de severidade ficaram próximos dos valores da severidade real, o que é observado quando são comparadas as linhas de regressão entre severidade real e estimada, sem escala (A-J e A*-J*) e com escala (K-T e K*-T*) (Figuras 2, 3, 4 e 5). Mesmo com o uso da escala, ainda ocorreram pequenas tendências de subestimação ou superestimação, no entanto, de forma aceitável, pois estudos demonstram que não só a boa qualidade de uma escala diagramática, mas também as estimativas dependem da percepção visual e da experiência de cada indivíduo na avaliação de doenças (SPÓSITO et al., 2004).

A precisão é um fator a ser considerado na validação de uma escala diagramática e é definida como a exatidão de uma operação na qual há rigor ou refinamento na medida. A precisão pode ser avaliada por meio do coeficiente de determinação da regressão, que deve ser próximo de $100 \%$, e pela variação dos erros absolutos (diferença entre severidade estimada e real). Com a utilização da escala diagramática proposta para mancha ocular em milho, os avaliadores apresentaram boa precisão, sendo que para os dois grupos de avaliadores (com experiência e sem experiência) ocorreu aumento do coeficiente de determinação de regressão quando se fez uso da escala

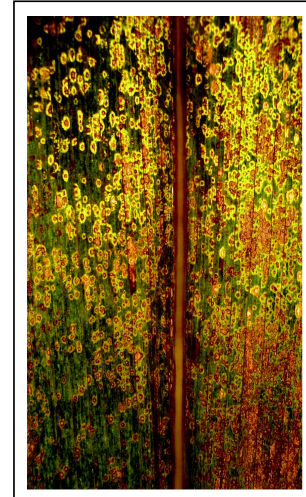

$51 \%$

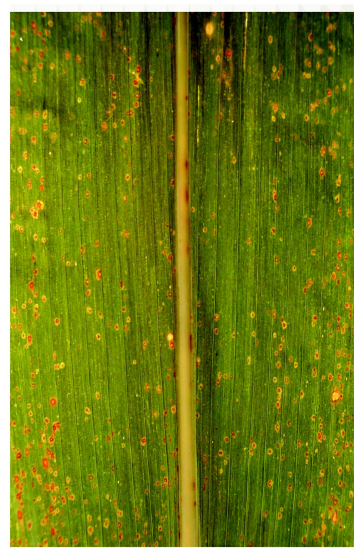

$4 \%$

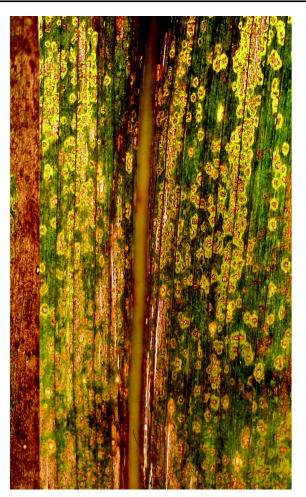

$32 \%$

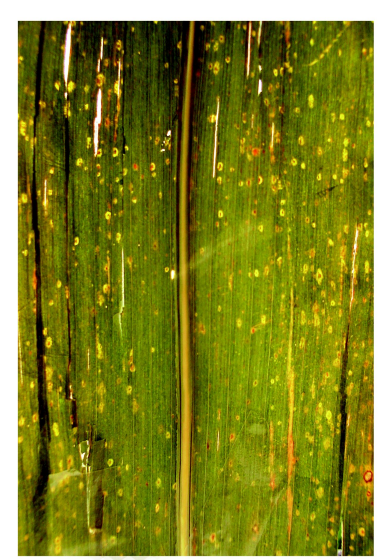

$2 \%$

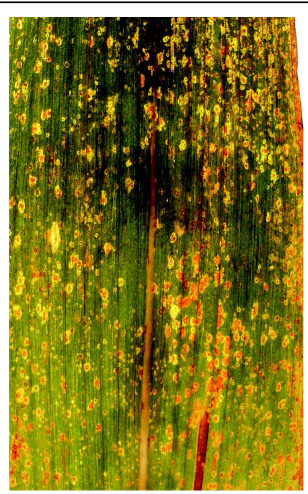

$18 \%$

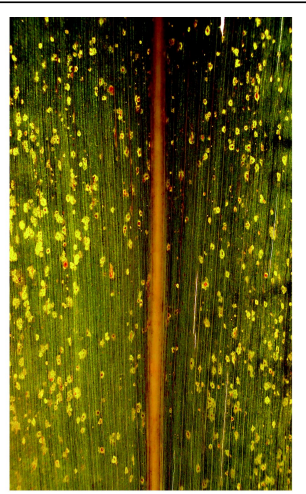

$9 \%$

Figura 1 - Escala diagramática para avaliação da severidade da mancha ocular em milho (Zea mays) causado por Kabatiella zeae. Valores em porcentagem da área foliar com sintomas. UTFPR - Campus Pato Branco, 2007. 


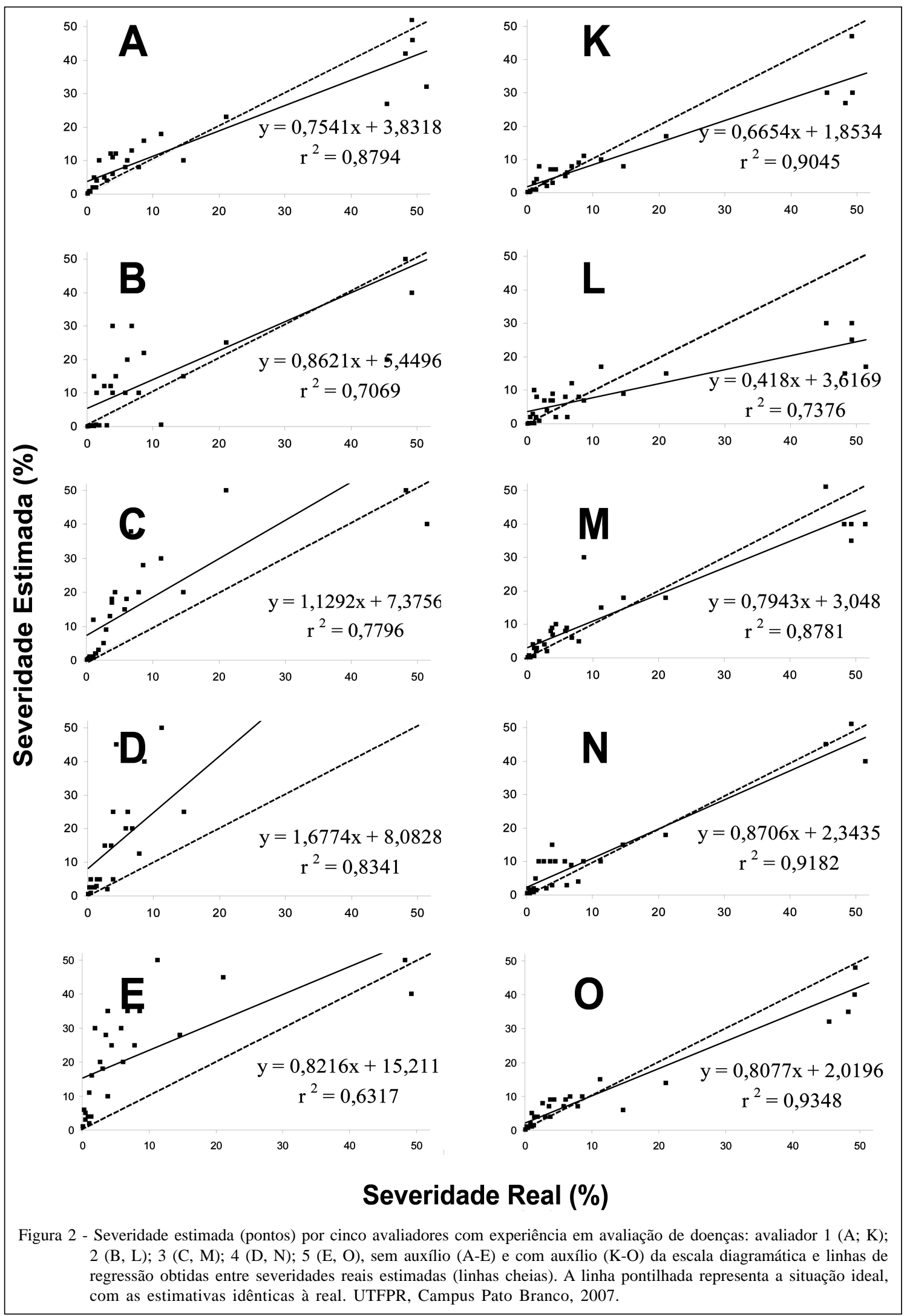

Ciência Rural, v.38, n.8, nov, 2008. 


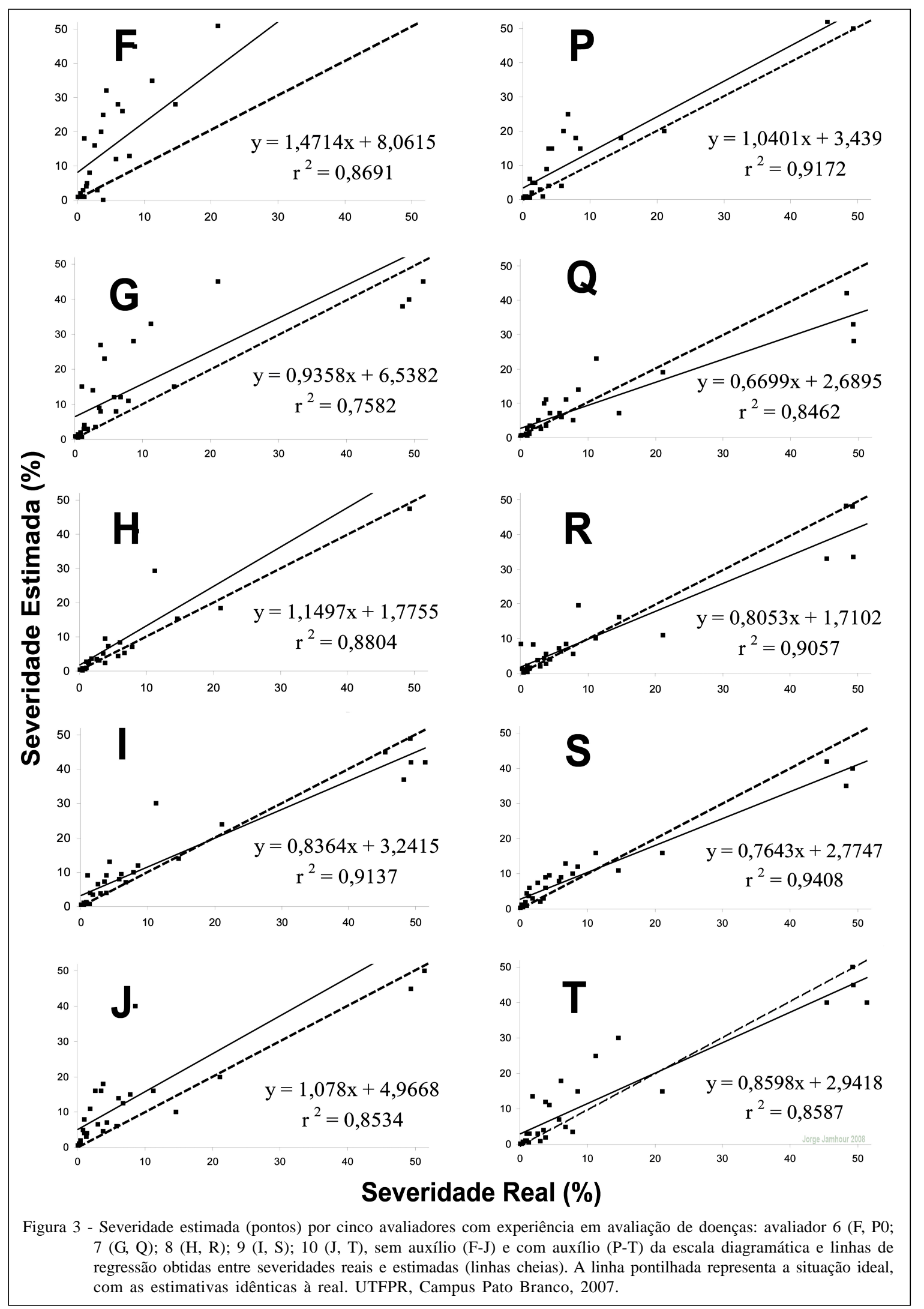

Ciência Rural, v.38, n.8, nov, 2008. 


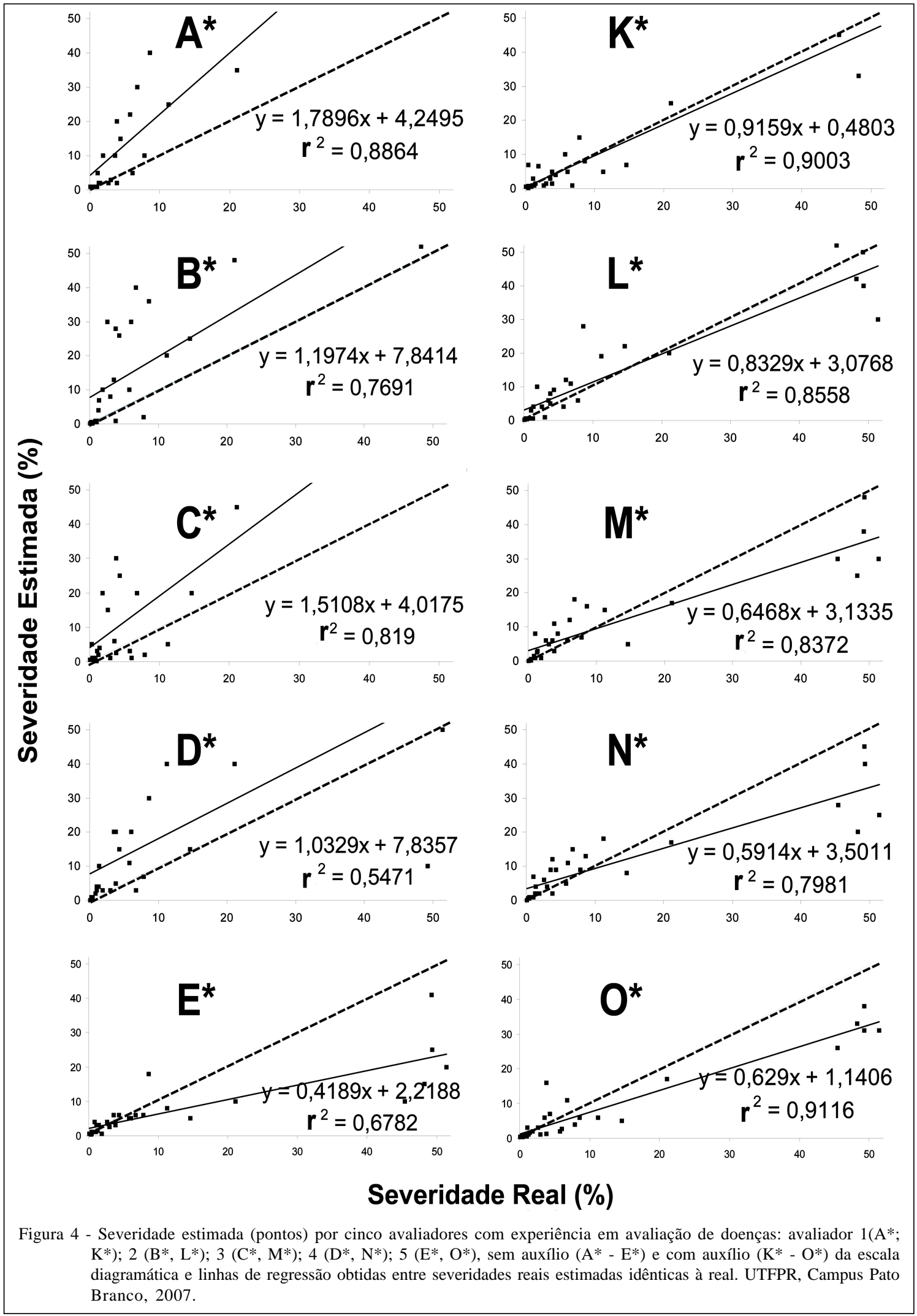

Ciência Rural, v.38, n.8, nov, 2008. 


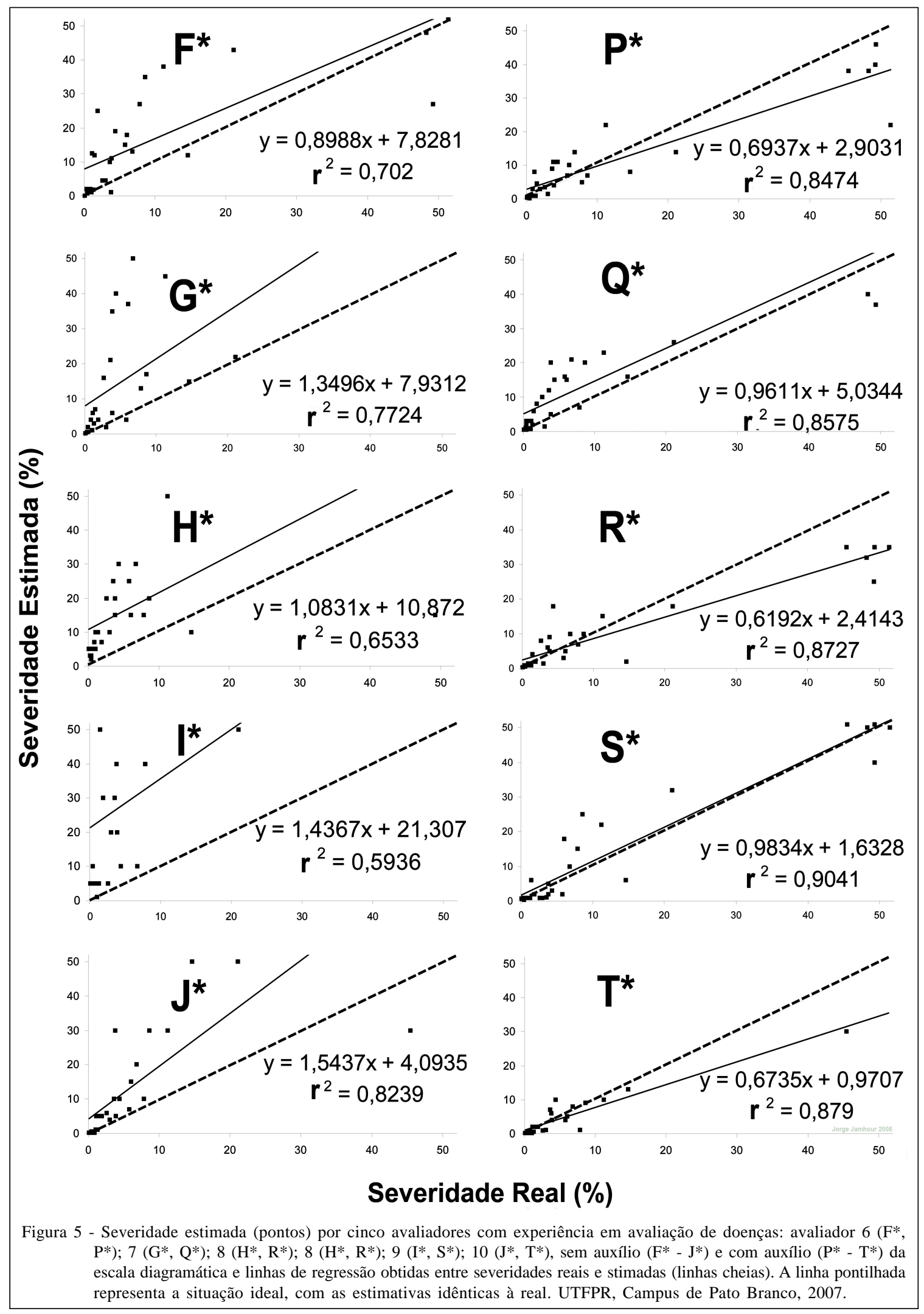

Ciência Rural, v.38, n.8, nov, 2008. 
(Figuras 2, 3, 4 e 5), indicando que, com o uso da escala, as estimativas foram sistematicamente relacionadas com o valor real.

Além do coeficiente de correlação, a boa precisão dos avaliadores pode ser observada com a determinação do erro absoluto ou do resíduo, ou seja, a diferença entre a severidade estimada e a real. Os valores obtidos para resíduos podem ser considerados adequados, pois, na maior parte, permaneceram dentro dos valores aceitáveis $(-10$ a +10$)$, segundo critérios adotados por programas de computadores para treinamento na quantificação de doenças como o Distrain (TOMERLIN \& HOWELL, 1988) e o Disease Pro (NUTTER JR \& WORAWITLIKIT, 1989). Quando foram comparados os grupos de avaliadores (sem escala e com escala), percebe-se que, de modo geral, o erro diminuiu para todos os avaliadores.

\section{CONCLUSÕES}

A utilização da escala diagramática para mancha ocular em milho permite quantificar os sintomas de forma acurada e precisa. O uso dessa escala em estudos epidemiológicos poderá proporcionar informações mais adequadas a respeito do patossistema Kabatiella zeae - milho.

\section{REFERÊNCIAS}

AGROCERES. Guia agroceres de sanidade. São Paulo: Sementes Agroceres, 1996. 72p.

BERGAMIN FILHO, A.; AMORIM, L. Doenças de plantas tropicais: epidemiologia e controle econômico. São Paulo: Agronômica Ceres, 1996. 299p.

CAMOCHENA, R.C. et al. Avaliação da sensibilidade de genótipos de milho à mancha ocular, causada por Kabatiella zeae. Fitopatologia Brasileira, v.32, p.225, 2007.
GOMES, A.M.A. et al. Elaboração e validação de escala diagramática para cercosporiose da alface. Summa Phytopathologica, v.30, p.38-42, 2004

HORSFALL, J.G.; COWLING, E.B. An advanced treatise-how disease develops in populations. Plant Disease. v.2, p.119136, 1978

HORSFALL, J.C.; BARRATT R.W. An improved grading system for measuring plant diseases. Phytopathology, v.35, p.665, 1945

LEITE, R.M.V.B.C.; AMORIM, L. Elaboração e validação de escala diagramática para mancha de Alternaria em girassol. Summa Phytopathologica, v.28, p.14-19, 2002.

MAZARO, S.M. et al. Escala diagramática para avaliação da severidade da mancha-de-micosferela em morangueiro. Ciência Rural, v.36, n.2, p.648-652, 2006.

NUTTER Jr., F.W.; WORAWITLIKIT, O. Disease Pro: a computer program for evaluating and improving a person's ability to assess disease proportion. Phytopathology, v.79, p.1135, 1989.

REIS, E.M.; CASA, R.T. Manual de identificação e controle de doenças de milho. Lavras: Ciências Agrotecnicas, 2004. p.325-331.

RODRIGUES, J.C.V. et al. Elaboração e validação de escala diagramática para leprose dos citros. Summa Phytopathologica, v.28, p.192-196, 2002.

SANTOS, I. dos et al. Ocorrência de mancha ocular em milho causada por Kabatiella zeae no Paraná e em Santa Catarina. Fitopatologia Brasileira, v.32, p.4, 2007.

SPÓSITO, M.B. et al. Elaboração e validação de escala diagramática para avaliação da severidade da mancha preta em frutos cítricos. Fitopatologia Brasileira, v.29, p.8185, 2004.

TOMERLIN, Jr.; HOWELL, T.A. Distrain: a computer program for training people to estimate disease severity on cereal leaves. Plant Disease, v.72, p.455-459, 1988. 